\title{
Surgical outcomes of perihilar cholangiocarcinoma based on the learning curve of a single surgeon at a tertiary academic hospital: A retrospective study
}

\author{
Hye Jeong Park, Dai Hoon Han, Gi Hong Choi, and Jin Sub Choi \\ Department of Surgery, Yonsei University College of Medicine, Seoul, Korea
}

\begin{abstract}
Backgrounds/Aims: Although it is difficult to master the surgical learning curve for treatment of perihilar cholangiocarcinoma (HCCA), there have been no studies on surgical outcomes between a novice and an experienced surgeon. Thus, the current study attempted to evaluate surgical outcomes from a single surgeon based on learning curve for surgical treatment of HCCA. Methods: From January 2008 to December 2016, a single surgeon performed surgical treatment for 108 patients with HCCA at Severance Hospital, Seoul, Korea. Among them, 101 patients with curative surgical resection were included in this study. The learning curve was assessed by a moving average graph and CUSUM method using operation time. Surgical outcomes between the early period group (EPG) and the late period group (LPG) were compared according to learning curve. Results: Operation time $(603.17 \pm 117.59$ and $432.03 \pm 91.77$ minutes; $p<0.001)$, amount of bleeding during operation $(1127.86 \pm 689.54$ and $613.05 \pm 548.31 \mathrm{ml} ; p<0.001)$, and severe complication rates $(47.6 \%$ and $27.1 \%, p=0.034)$ were significantly smaller in the LPG. There was no significant difference in R0 resection rate $(85.7 \%$ and $76.3 \% ; p=0.241)$ as well as long-term survival rate. Conclusions: In this study, operation time, amount of bleeding during operation, length of hospital stay, and severe complication rate were improved after stabilization of the learning curve. However, RO resection rate and survival outcomes were not significantly influenced by the learning curve for surgical treatment of HCCA. (Ann Hepatobiliary Pancreat Surg 2021;25:54-61)
\end{abstract}

Key Words: Perihilar cholangiocarcinoma; Learning curve; Surgical outcome

\section{INTRODUCTION}

Although perihilar cholangiocarcinoma (HCCA) represents $50-70 \%$ of all types of bile duct carcinomas, it is a very rare disease, with an annual incidence rate no greater than $1: 100,000 .{ }^{1}$ Radical surgery with negative histological margins is needed for long-term survival. ${ }^{2}$ However, the adjacent location of HCCA to the hepatic artery, portal vein, and hepatic parenchyma complicates complete resection. Moreover, radical surgery for HCCA includes liver resection, bilio-enteric reconstruction, and radical lymph node dissection around the perihilar, retropancreatic, and para-aortic areas. Thus, infrequent occurrence and demanding surgical techniques may complicate the learning curve of surgical expertise for treatment of HCCA. $^{3}$
Therefore, the current study set out to determine the learning curve of radical surgery for HCCA in a single surgeon working in a tertiary academic hospital and to assess the perioperative outcomes according to surgical proficiency.

\section{MATERIALS AND METHODS}

\section{Patients}

From January 2008 to December 2016, a total of 108 consecutive patients underwent curative-intent surgery for HCCA by a single surgeon (CGH) at Yonsei University College of Medicine, Seoul, Korea. Excluding seven cases (one case with only laparotomy, three cases with hepatopancreaticoduodenectomy, one case with segmental resection of the bile duct, and two cases with benign disease

Received: August 31, 2020; Revised: October 10, 2020; Accepted: October 11, 2020

Corresponding author: Dai Hoon Han

Department of Surgery, Yonsei University College of Medicine, 50 Yonsei-ro, Seodaemoon-gu, Seoul 03722, Korea Tel: +82-2-2228-2139, Fax: +82-2-313-8289, E-mail: dhhan@yuhs.ac

Copyright (C) 2021 by The Korean Association of Hepato-Biliary-Pancreatic Surgery

This is an Open Access article distributed under the terms of the Creative Commons Attribution Non-Commercial License (http://creativecommons.org/ censes/by-nc/4.0) which permits unrestricted non-commercial use, distribution, and reproduction in any medium, provided the original work is properly cited. Annals of Hepato-Biliary-Pancreatic Surgery • pISSN: 2508-5778 • elSSN: 2508-5859 
on final pathology report), 101 patients who underwent curative surgery consisting of liver resection, radical lymph node dissection, and Roux-en-Y hepaticojejunostomy for HCCA were included in this study. Therefore, a total of 101 patients was divided into two groups according to learning curve analysis: the early period group (EPG), consisting of 42 patients, and the late period group (LPG), consisting of 59 patients (Fig. 1).

\section{Learning curve analysis}

Operative time was used as a variable for learning outcome in this study and was defined as incision-to-closure time. Although the surgical procedure of HCCA was diverse and complex, it is composed of three essential processes: radical lymph node dissection, liver resection, and bilio-enteric anastomosis. Among these three steps, procedural time for liver resection may be affected by extent of the resection plane. Theoretically, central bisectionectomy or anterior sectionectomy might require more than twice the effort for parenchymal dissection compared to a right hemihepatectomy, left hemihepatectomy, right trisectionectomy, or left trisectionectomy, which only need one-side resection of the liver. Moreover, vascular resection and reconstruction to remove the tumor-invaded hepatic artery or portal vein may also prolong the operation time. Therefore, 71 patients who underwent one-side liver resection (hemihepatectomy, extended hemihepatectomy, and trisectionectomy) without vascular resection and anastomosis were selected for learning curve analysis. The learning curve was assessed with the cumulative sum (CUSUM) method and a moving average graph.

\section{CUSUM method}

The CUSUM method is a descriptive method that can represent data trends by calculating the serial differences between raw data and the mean value. ${ }^{4}$ In this study, 71 cases were ordered chronologically from the first to the last, and the CUSUM of the operation time $\left(\mathrm{CUSUM}_{\mathrm{OT}}\right)$ was defined as CUSUM $M_{O T}=\sum_{i=1}^{n}\left(O T_{\text {mean }}-O T_{i}\right)$, where $O T_{i}$ is individual operation time, and $O T_{\text {mean }}$ is the mean operation time of the 71 cases. First, CUSUM was obtained by summing the differences between mean operative time and serial operative time. The learning curve was determined by plotting CUSUM

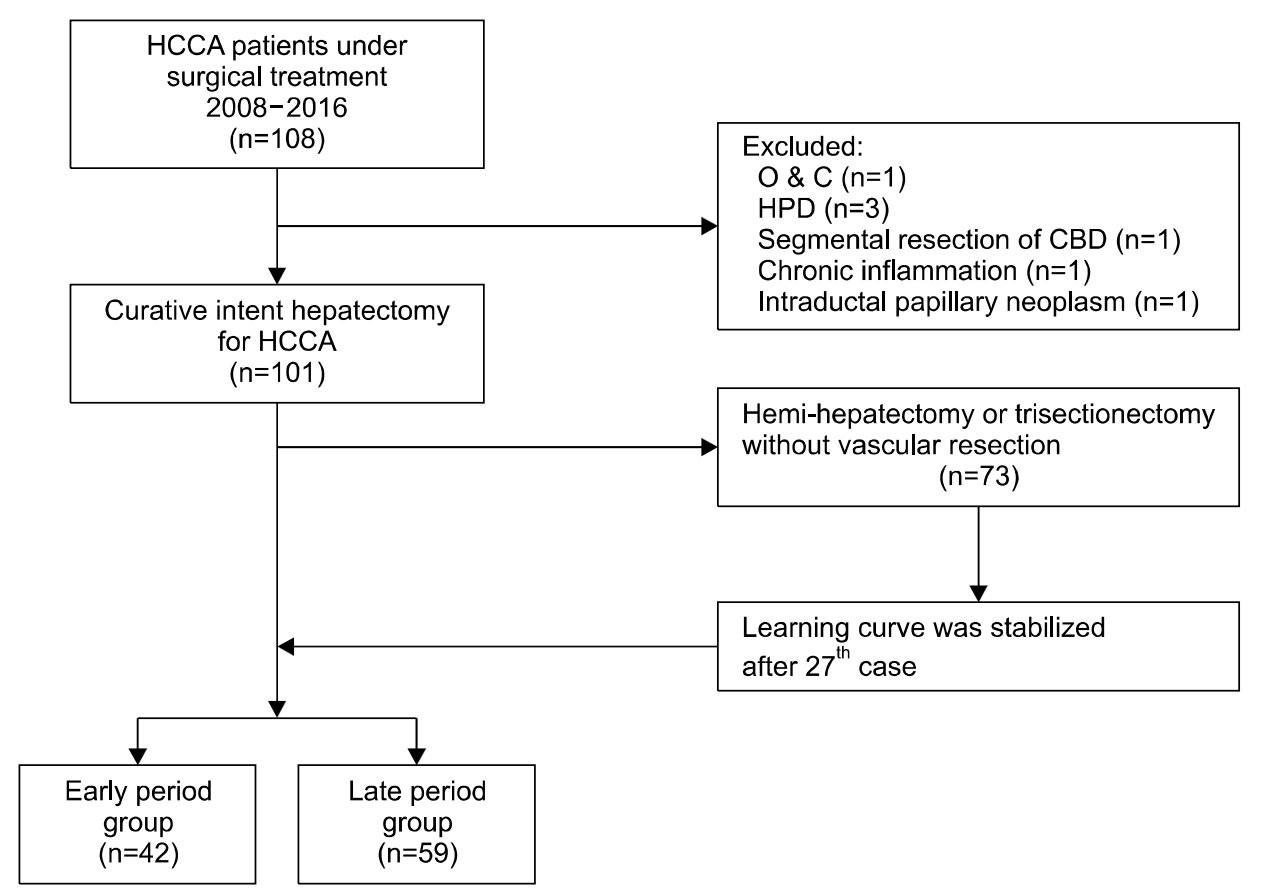

Fig. 1. Flow diagram of study design. For the study, 101 of 108 consecutive patients who underwent surgical treatment for HCCA were selected. Of these, 73 patients with one-side liver resection without vascular resection were selected to evaluate the learning curve. The $27^{\text {th }}$ of 73 selected patients was the first test patient after stabilization of the learning curve. This $27^{\text {th }}$ patient was matched to the $43^{\text {rd }}$ consecutive of 101 patients. Thus, the study group was subdivided into the early period group $(n=42)$ and the late period group $(n=59)$. 


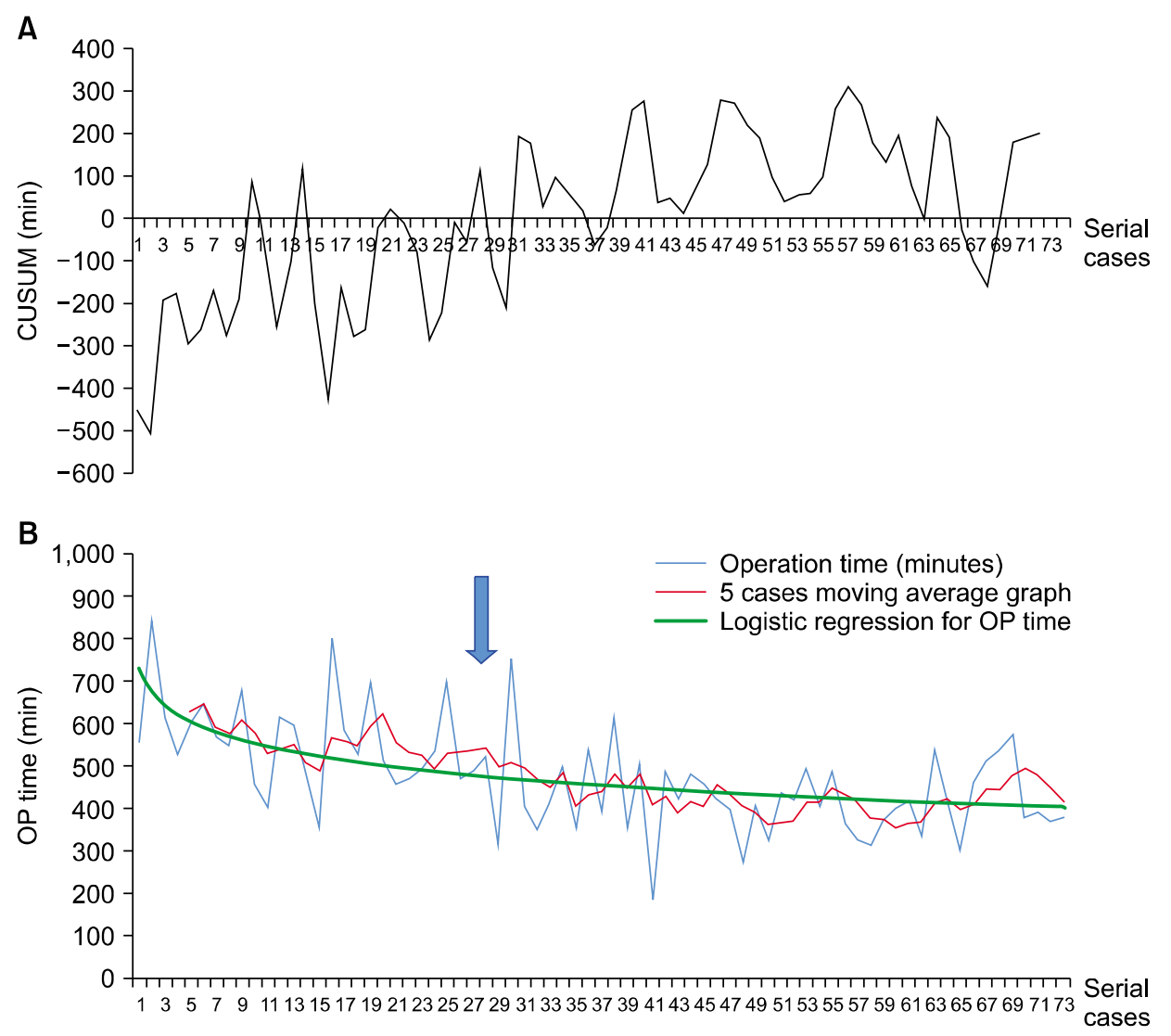

Fig. 2. CUSUM and moving average graph by operation time for evaluating the learning curve. (A) CUSUM with operation time for 73 consecutive patients with hemi-hepatectomy or trisectionectomy without vascular resection. A CUSUM=0 meant the operation time was the same as the mean operation time. A positive CUSUM meant the operation time was shorter than the mean operation time. The operation times were steadily shorter than the mean operation time after the $27^{\text {th }}$ case. (B) Moving average graph for each of five serial cases. A logistic regression graph was drawn to identify the learning achievement point. The steep slope of the logistic regression graph before the $27^{\text {th }}$ case started to flatten after the $27^{\text {th }}$ case. Thus, the learning curve may be stabilized after the $27^{\text {th }}$ of 73 consecutive patients.

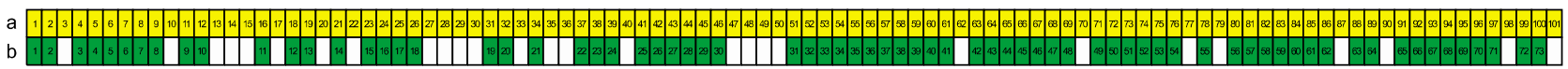
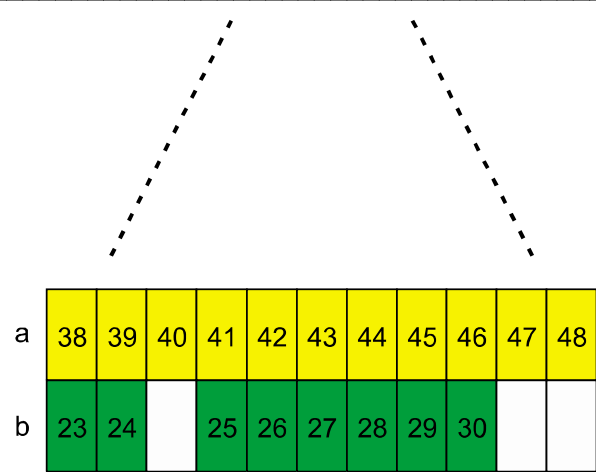

Fig. 3. Early versus late period group assignment according to case-matching. The $27^{\text {th }}$ of 73 patients with a one-side hepatectomy without vascular resection (b, green bar) was matched to the $43^{\text {rd }}$ of 101 consecutive patients. The first 42 patients were classified as the early period group, and the subsequent 59 patients were classified as the late period group. 
of zero meant that $O T_{i}$ was the same as $O T_{\text {mean }}$. Thus,

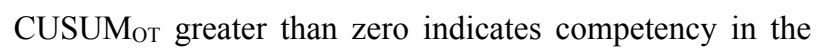
procedure. The CUSUM zero baseline after the $27^{\text {th }}$ case.

\section{Verification of the learning curve with a moving average graph}

The mean operation time for each of five sequential cases was calculated and plotted. The moving average graph converged toward a plateau after the $27^{\text {th }}$ case. A logarithm graph for serial operation time was created using Microsoft EXCEL 2016 (Microsoft Inc., Redmond, WA, USA) with the formula $y=-93.43 \ln (x)+786.28$, which also showed a plateau after 27 cases (Fig. 2B). Therefore, procedure competence was stabilized after the $27^{\text {th }}$ of 71 cases selected for this learning curve analysis. The $27^{\text {th }}$ case in these 71 selected patients was the $43^{\text {rd }}$ of 101 sequential patients (Fig. 3). Therefore, the patients were di- chotomized into two groups of EPG $(\mathrm{n}=42)$ and LPG $(\mathrm{n}=59)$.

\section{Statistical analysis}

Normality of continuous variables was tested using Levene's test. Continuous variables with symmetrical distribution were compared using the independent $t$-test and presented as mean with standard deviation. Continuous data with asymmetrical distribution were compared using Mann-Whitney $U$ test and described as median value with interquartile range. Categorical variables were compared with the $\chi^{2}$ or Fisher's exact test as appropriate. Survival was calculated using the Kaplan-Meier method and compared between groups using the log-rank test. Perioperative mortality was included in overall survival analysis but excluded from disease-free survival analysis. Cox regression model with forward stepwise logistic regression analysis was applied for multivariate analysis using sig-

Table 1. Patient characteristics

\begin{tabular}{|c|c|c|c|}
\hline Variables & EPG $(n=42)$ & LPG $(n=59)$ & $p$-value \\
\hline Sex (male:female) & $24: 18$ & $41: 18$ & 0.202 \\
\hline Age & $62.29 \pm 10.59$ & $66.00 \pm 9.99$ & 0.075 \\
\hline \multicolumn{4}{|l|}{ Pre-OP laboratory findings } \\
\hline Albumin (g/dl) & $3.53 \pm 0.44$ & $3.55 \pm 0.45$ & 0.845 \\
\hline AST (IU/L) & $42.36 \pm 28.37$ & $39.97 \pm 23.20$ & 0.643 \\
\hline ALT (IU/L) & $35.79 \pm 25.81$ & $45.44 \pm 40.64$ & 0.178 \\
\hline GGT (IU/L) & $445.98 \pm 453.69$ & $344.15 \pm 291.17$ & 0.205 \\
\hline CEA (ng/ml) & $4.13 \pm 5.87$ & $3.82 \pm 5.66$ & 0.969 \\
\hline CA $19-9(\mathrm{U} / \mathrm{ml})$ & $1166.77 \pm 3339.59$ & $468.03 \pm 1071.22$ & 0.197 \\
\hline Total bilirubin $(\mathrm{mg} / \mathrm{dl})$ & $1.69 \pm 1.08$ & $1.36 \pm 0.79$ & 0.099 \\
\hline Highest total bilirubin $(\mathrm{mg} / \mathrm{dl})$ & $8.33 \pm 6.30$ & $6.87 \pm 6.53$ & 0.266 \\
\hline Neoadjuvant CCRT & $6(14.3 \%)$ & $26(44.1 \%)$ & 0.002 \\
\hline PVE & $3(7.1 \%)$ & $14(23.7 \%)$ & 0.028 \\
\hline \multicolumn{4}{|l|}{ Bismuth-Corlette type } \\
\hline I & $3(7.1 \%)$ & $2(3.4 \%)$ & \\
\hline II & $8(19.0 \%)$ & $8(13.6 \%)$ & \\
\hline IIIa & $8(19.0 \%)$ & $26(44.1 \%)$ & \\
\hline IIIb & $4(9.5 \%)$ & $9(15.3 \%)$ & \\
\hline IV & $19(45.2 \%)$ & $14(23.7 \%)$ & \\
\hline \multicolumn{4}{|l|}{ AJCC stage } \\
\hline 0 & $1(2.4 \%)$ & $2(3.4 \%)$ & \\
\hline I & $1(2.4 \%)$ & $2(3.4 \%)$ & \\
\hline II & $20(47.6 \%)$ & $28(47.5 \%)$ & \\
\hline IIIA & $0(0 \%)$ & $7(11.9 \%)$ & \\
\hline IIIB & $18(42.9 \%)$ & $17(28.8 \%)$ & \\
\hline IVB & $2(4.8 \%)$ & $3(5.1 \%)$ & \\
\hline
\end{tabular}

EPG, early period group; LPG, late period group; AST, aspartate aminotransferase; ALT, alanine aminotransferase; GGT, gamma-glutamyltransferase; CEA, carcinoembryonic antigen; CA 19-9, carbohydrate antigen 19-9; CCRT, concurrent chemo-radiation therapy; PVE, portal vein embolization; AJCC, American Joint Committee on Cancer; LN, lymph node 
nificant univariate variables affecting survival. Statistical analyses were performed using SPSS 25 for Windows (SPSS Inc., Chicago, IL, USA). A value of $p<0.05$ was considered statistically significant.

\section{RESULTS}

\section{Patient characteristics}

Of the 101 patients, 65 were men (64.4\%), and the mean age at operation was $64.46 \pm 10.36$ years. A total of 32 patients received neoadjuvant chemotherapy (31.7\%), and 17 patients underwent preoperative portal vein embolization (16.8\%). Bismuth-Corlette type IIIa was the most common type of HCCA $(n=34,33.7 \%)$. Right hemi-hepatectomy $(n=41,40.6 \%)$ was performed most frequently, followed by left hemihepatectomy $(n=32,31.7 \%)$. The most common pathologic stage according to AJCC $8^{\text {th }}$ edition was stage II $(n=48,47.5 \%)$, followed by stage IIIB $(\mathrm{n}=35,34.7 \%)$. Of all patients, 40 had $\mathrm{LN}$ metastasis (39.6\%), and the R0 resection rate was $80.2 \%(n=81)$.

\section{Perioperative outcomes of early and late period groups}

There was no significant difference in sex, age, or preoperative laboratory findings between the two groups. However, patients with complicated preoperative proce- dures such as neoadjuvant concurrent chemo-radiation therapy (CCRT) and portal vein embolization (PVE) were more common in the LPG (Table 1). Bismuth-Corlette type IV was most common among EPG patients $(n=19$, $45.2 \%$ ), while type IIIa was most common in the LPG $(n=26,44.1 \%)$, though there was no statistically significant difference between the two groups. AJCC stage II was most common in both groups (EPG: $n=20,47.6 \%$, LPG: $n=28,47.5 \%$ ).

In terms of type of liver resection, left hemihepatectomy in the EPG $(n=13,31.0 \%)$ and right hemihepatectomy in the LPG $(n=30,50.8 \%)$ were most frequently performed. There was no significant difference in LN metastasis rate, $\mathrm{R} 0$ resection rates or number of retrieved lymph nodes during operation between the two groups (Table 2). However, perioperative outcomes were improved in the LPG, with significantly shorter average operation time (603.17 \pm 117.59 vs. $432.03 \pm 91.77, p<0.001)$, significantly decreased bleeding during operation (1127.86 \pm $689.54 \mathrm{ml}$ vs. $613.05 \pm 548.31 \mathrm{ml}, p<0.001$ ), and significantly decreased length of hospital stay (median hospital stay day: 23 days vs. 18 days, $p=0.025$ ). Severe complications greater than grade IIIA according to ClavienDindo classification were significantly more frequent in the EPG (47.6\% vs. $27.1 \%, p=0.034)$. However, there was no significant difference in overall complications rate or

Table 2. Perioperative outcomes

\begin{tabular}{lccc}
\hline \multicolumn{1}{c}{ Variables } & EPG $(\mathrm{n}=42)$ & LPG $(\mathrm{n}=59)$ & $p$-value \\
\hline Liver resection type & & & $30(50.8 \%)$ \\
Rt. hemihepatectomy & $11(26.2 \%)$ & $19(32.2 \%)$ \\
Lt. hemihepatectomy & $13(31.0 \%)$ & $3(5.1 \%)$ & $0(0 \%)$ \\
Rt. trisectionectomy & $1(2.4 \%)$ & $1(1.7 \%)$ & 0.071 \\
Lt. trisectionectomy & $8(19.0 \%)$ & $5(8.5 \%)$ & 0.241 \\
Caudate lobectomy & $0(0 \%)$ & $1(1.7 \%)$ & 0.434 \\
Central bisectionectomy & $2(4.8 \%)$ & $0(0 \%)$ & $<0.001$ \\
Anterior sectionectomy & $1(2.4 \%)$ & $19(32.2 \%)$ & $<0.001$ \\
Segmentectomy 4 & $6(14.3 \%)$ & $45(76.3 \%)$ & 0.025 \\
LN metastasis & $21(50.0 \%)$ & $52(88.1 \%)$ & 0.317 \\
R0 resection & $36(85.7 \%)$ & $432.03 \pm 91.77$ & 0.034 \\
LN retrieval $\geq 4$ & $39(92.9 \%)$ & $613.05 \pm 548.31$ & 0.166 \\
Operation time (minutes) & $603.17 \pm 117.59$ & $18(12.25-23)$ & \\
Bleeding amount during operation (ml) & $1127.86 \pm 689.54$ & $38(64.4 \%)$ & \\
Length of hospital stay (day) & $23(16-30)$ & $16(27.1 \%)$ & $(1.7 \%)$ \\
Overall complications & $31(73.8 \%)$ & & \\
Severe complications & $20(47.6 \%)$ & $3(7.1 \%)$ & \\
Perioperative mortality & & & \\
\hline
\end{tabular}

EPG, early period group; LPG, late period group; Rt., right; Lt., left; LN, lymph node 

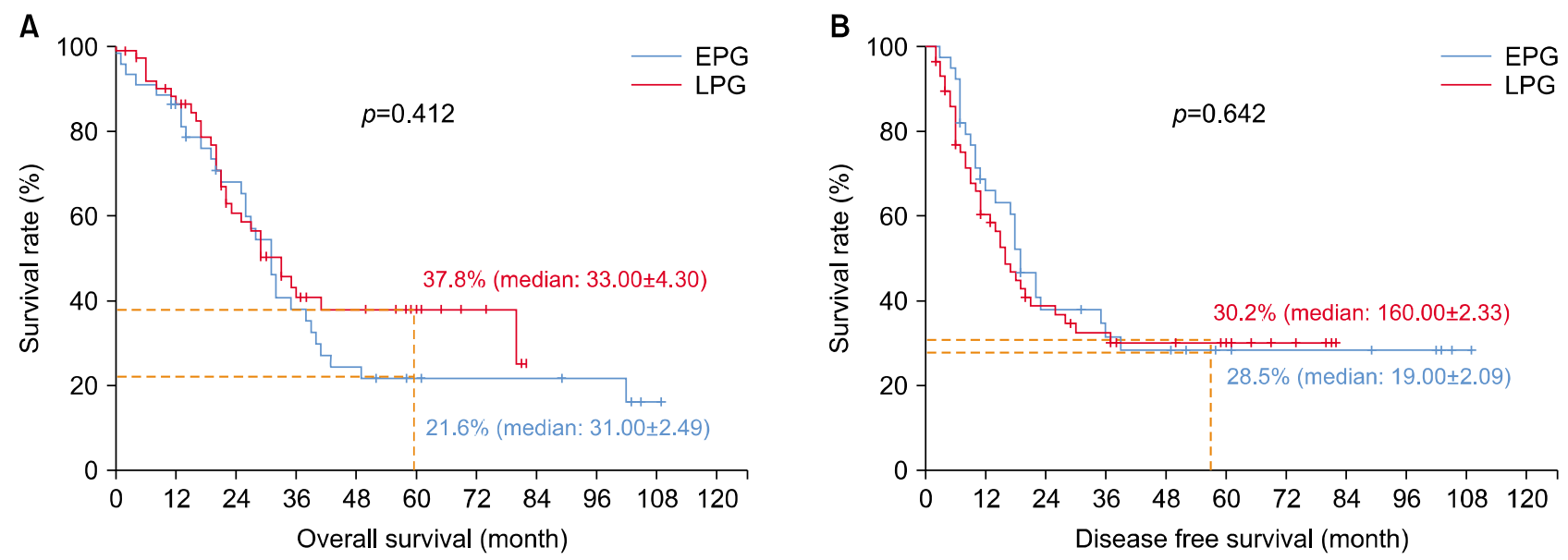

Fig. 4. Comparison Overall survival and Disease free survival graph between two groups. (A) The 5-year overall survival rate was $21.6 \%$ and $37.8 \%$ in the EPG and LPG, respectively $(p=0.412)$. (B) The 5 -year disease free survival rate was $28.5 \%$ and $30.2 \%$ for the EPG and LPG, respectively $(p=0.642)$.

Table 3. Disease-free survival and overall survival with multivariate analysis

\begin{tabular}{ccccc}
\hline Variables & Coefficient & Standard error & $p$-value & Relative risk (95\% CI) \\
\hline Disease-free survival & & & & \\
Pre-OP CEA & 0.895 & 0.308 & 0.004 & $2.139-2.755$ \\
LN metastasis & 0.502 & 0.254 & 0.048 & $1.398-1.906$ \\
Overall survival & & & & \\
Pre-op serum albumin & 0.741 & 0.258 & 0.004 & $1.840-2.356$ \\
Pre-OP CEA & 0.668 & 0.322 & 0.038 & $1.629-2.273$ \\
\hline
\end{tabular}

LN, lymph node; Pre-op, preoperative; CEA, carcinoembryonic antigen

perioperative mortality between the two groups (Table 2).

Regarding severe complications, cases of intra-abdominal fluid collection, pleural effusion, and bile leakage lead to percutaneous catheter drainage, and wound infection required secondary wound closure in grade IIIA complications. Patients with grade IV complications were admitted to intensive care units for post-operative bleeding, cholangitis, or intestinal obstruction. Biliary sepsis and aspiration pneumonia were the causes of perioperative mortality.

\section{Survival outcomes}

There was no significant difference in disease-free survival (DFS) or overall survival (OVS) rate between the two groups (Fig. 4). The 5-year DFS rate was $28.5 \%$ and $30.2 \%$ for the EPG and LPG, respectively ( $p=0.642$ ). The 5 -year OVS rate was $21.6 \%$ and $37.8 \%$ in the EPG and LPG, respectively $(p=0.412)$. Preoperative serum carcinoembryonic antigen (CEA) level over $5 \mathrm{ng} / \mathrm{ml}$ and meta- stasis to lymph node were te significant risk factors for poor DFS rates according to multivariate analysis. The significant poor prognostic factors for OVS rates were high preoperative CEA level over $5 \mathrm{ng} / \mathrm{ml}$ and low serum albumin level under $3.5 \mathrm{~g} / \mathrm{dl}$ (Table 3 ).

\section{DISCUSSION}

HCCA is a very rare disease, and only one-quarter of all patients are suitable for curative resection. ${ }^{5}$ Thus, it is very difficult to achieve surgical proficiency for HCCA treatment in a short period of time. Moreover, curative-intent operations for HCCA include various complicated surgical procedures. ${ }^{6,7}$ Along with dismal long-term oncologic outcomes even after curative surgery, perioperative outcomes are relatively poor. ${ }^{8,9}$ Therefore, such surgical treatment for patients with HCCA is typically performed in tertiary care hospitals. However, there is lack of information regarding the impact of surgical experience on 
surgical outcome for HCCA. To the best of our knowledge, the present study is the first learning curve study on surgical treatment of HCCA in a tertiary academic hospital.

Various surgical factors, such as operation time and intraoperative blood loss, and dichotomous surgical variables including postoperative complication or mortality may represent learning outcomes. Among these variables, operation time is the most commonly used variable for learning outcomes due to its easy accessibility even in retrospective studies. CUSUM analysis based on operation time may effectively reduce the inherent variability of surgical data and improve understanding of the learning curve. ${ }^{10}$ The current study also used operation time as a learning outcome. However, complex procedures such as central bisectionectomy or segmental resection of the portal vein or hepatic artery might affect the operation time. ${ }^{2}$ Therefore, the learning curve was created using data from patients undergoing similar procedures: one-side hepatectomy without vascular resection and anastomosis. Indeed, perioperative outcomes of the LPG were significantly improved compared to those of the EPG. Thus, the current learning curve study appears to reasonable to distinguish an inexperienced period from an experienced period. Indication of the operation was also extended after achieving learning curve. There were significantly more patients with neoadjuvant chemo-radiation therapy or portal vein embolization in the LPG (Table 1). ${ }^{2,11}$ However, severe complications, operation time, amount of bleeding during operation, and length of hospital stay were significantly reduced in the LPG.

Although the perioperative outcome was significantly improved in the LPG, there was no difference in longterm survival outcome between the LPG and EPG. There were no significant differences in disease-free and overall survival rates between the two groups, even after considering cancer stage in subgroup analyses. In general, the 5-year survival rates were around 30\% even after curative resection due to the high chance of recurrence. Even though a few studies have reported that perioperative outcomes such as perioperative blood transfusion were poor prognostic factors for long-term survival, ${ }^{12}$ most studies have revealed tumor-related factors such as lymph node metastasis, R1 resection, high preoperative carcinoembryonic antigen level, poor differentiation, and vascular invasion as independent risk factors for long-term survival after curative treatment of HCCA.,12-21 Therefore, improved perioperative outcomes might not necessarily improve the long-term survival outcomes.

This study has some limitations. This was a single-institutional, retrospective study involving a single surgeon. Although more than 100 cases were included, which might be considered a high volume given the rarity of $\mathrm{HCCA}^{22}$ it is difficult to generalize the results that 40 cases are necessary to achieve surgical proficiency for treatment of HCCA. Therefore, prospective multi-center studies for novice surgeons are needed.

In conclusion, operation time, amount of bleeding during operation, length of hospital stay, and complication rates were improved after stabilization of the surgical learning curve. However, R0 resection rates and survival outcomes were not significantly influenced by the learning curve for surgical treatment of HCCA.

\section{ORCID}

Hye Jeong Park: https://orcid.org/0000-0002-1487-8899

Dai Hoon Han: https://orcid.org/0000-0003-2787-7876

Gi Hong Choi: https://orcid.org/0000-0002-1593-3773

Jin Sub Choi: https://orcid.org/0000-0002-6467-6494

\section{AUTHOR CONTRIBUTIONS}

HJP: Validation, Formal Analysis, Investigation, Writing Original draft, Writing-Review \& Editing, Visualization, Project Administration. Conceptualization, Resources, Methodology, Writing-review \& Editing, Supervision: DHH. Conceptualization, Resources, Investigation, Writing-review \& Editing: GHC. Investigation, Resources, Writing-Review \& editing: JSC.

\section{REFERENCES}

1. Zhang X, Liu H. Klatskin tumor: a population-based study of incidence and survival. Med Sci Monit 2019;25:4503-4512.

2. Mizuno T, Ebata T, Nagino M. Advanced hilar cholangiocarcinoma: an aggressive surgical approach for the treatment of advanced hilar cholangiocarcinoma: perioperative management, extended procedures, and multidisciplinary approaches. Surg Oncol 2020;33:201-206.

3. Sakata J, Ebata T, Wakai T. Evolution of radical resection for perihilar cholangiocarcinoma. J Hepatobiliary Pancreat Sci 2018; 25:249-251. 
4. Wang M, Meng L, Cai Y, Li Y, Wang X, Zhang Z, et al. Learning curve for laparoscopic pancreaticoduodenectomy: a CUSUM analysis. J Gastrointest Surg 2016;20:924-935.

5. Olthof PB, Miyasaka M, Koerkamp BG, Wiggers JK, Jarnagin WR, Noji T, et al. A comparison of treatment and outcomes of perihilar cholangiocarcinoma between Eastern and Western centers. HPB (Oxford) 2019;21:345-351.

6. Ratti F, Cipriani F, Fiorentini G, Hidalgo Salinas C, Catena M, Paganelli $\mathrm{M}$, et al. Management of hilum infiltrating tumors of the liver: the impact of experience and standardization on outcome. Dig Liver Dis 2019;51:135-141.

7. Peng DZ, Lu J, Li B, Hu HJ, Ye XW, Xiong XZ, et al. A simple scoring system to predict early recurrence of Bismuth-Corlette type IV perihilar cholangiocarcinoma. Gastroenterol Rep (Oxf) 2019; 7:345-353.

8. Tran TB, Ethun CG, Pawlik TM, Schmidt C, Beal EW, Fields $\mathrm{RC}$, et al. Actual 5-year survivors after surgical resection of hilar cholangiocarcinoma. Ann Surg Oncol 2019;26:611-618.

9. Juntermanns B, Kaiser GM, Reis H, Gries S, Kasper S, Paul A, et al. Long-term survival after resection for perihilar cholangiocarcinoma: impact of UICC staging and surgical procedure. Turk J Gastroenterol 2019;30:454-460.

10. Valsamis EM, Chouari T, O'Dowd-Booth C, Rogers B, Ricketts D. Learning curves in surgery: variables, analysis and applications. Postgrad Med J 2018;94:525-530.

11. Matsuyama R, Morioka D, Mori R, Yabushita Y, Hiratani S, Ota $\mathrm{Y}$, et al. Our rationale of initiating neoadjuvant chemotherapy for hilar cholangiocarcinoma: a proposal of criteria for "borderline resectable" in the field of surgery for hilar cholangiocarcinoma. World J Surg 2019;43:1094-1104.

12. Kimura N, Young AL, Toyoki Y, Wyatt JI, Toogood GJ, Hidalgo E, et al. Radical operation for hilar cholangiocarcinoma in comparable Eastern and Western centers: outcome analysis and prognostic factors. Surgery 2017;162:500-514.

13. Gao Y, Xu D, Wu YS, Chen D, Xiong W. Increasing negative lymph node count is independently associated with improved long-term survival in resectable perihilar cholangiocarcinomas. Medicine (Baltimore) 2019;98:e14943.

14. Gaspersz MP, Buettner S, Roos E, van Vugt JLA, Coelen RJS, Vugts $\mathrm{J}$, et al. A preoperative prognostic model to predict surgical success in patients with perihilar cholangiocarcinoma. J Surg Oncol 2018;118:469-476.

15. He M, Xu X, Feng H, Chen W, Liu H, Zhang Y, et al. Regional lymphadenectomy vs. extended lymphadenectomy for hilar cholangiocarcinoma (Relay-HC trial): study protocol for a prospective, multicenter, randomized controlled trial. Trials 2019; 20:528.

16. Higuchi R, Yazawa $\mathrm{T}$, Uemura S, Izumo W, Ota T, Kiyohara $\mathrm{K}$, et al. Surgical outcomes for perihilar cholangiocarcinoma with vascular invasion. J Gastrointest Surg 2019;23:1443-1453.

17. Kambakamba P, Linecker M, Slankamenac K, DeOliveira ML. Lymph node dissection in resectable perihilar cholangiocarcinoma: a systematic review. Am J Surg 2015;210:694-701.

18. Komaya K, Ebata T, Yokoyama Y, Igami T, Sugawara G, Mizuno $\mathrm{T}$, et al. Recurrence after curative-intent resection of perihilar cholangiocarcinoma: analysis of a large cohort with a close postoperative follow-up approach. Surgery 2018;163:732-738.

19. Lin $\mathrm{H}, \mathrm{Wu} \mathrm{YS}, \mathrm{Li} \mathrm{Z}$, Jiang Y. Prognostic value of retrieved lymph node counts in patients with node-negative perihilar cholangiocarcinomas. ANZ J Surg 2018;88:E829-E834.

20. Lurje G, Bednarsch J, Czigany Z, Lurje I, Schlebusch IK, Boecker J, et al. The prognostic role of lymphovascular invasion and lymph node metastasis in perihilar and intrahepatic cholangiocarcinoma. Eur J Surg Oncol 2019;45:1468-1478.

21. Qi F, Zhou B, Xia J. Nomograms predict survival outcome of Klatskin tumors patients. PeerJ 2020;8:e8570.

22. Chaudhary RJ, Higuchi R, Nagino M, Unno M, Ohtsuka M, Endo I, et al. Survey of preoperative management protocol for perihilar cholangiocarcinoma at 10 Japanese high-volume centers with a combined experience of 2,778 cases. J Hepatobiliary Pancreat Sci 2019;26:490-502. 